\title{
A APLICAÇÃO DA PROGRAMAÇÃO INTEIRA NA SOLUÇÃO LOGÍSTICA DO TRANSPORTE DE CARGA: O SOLVER E SUAS LIMITAÇṌES NA BUSCA PELA SOLUÇÃO ÓTIMA
}

\section{APPLICATION OF INTEGER PROGRAMMING ON LOGISTICS SOLUTION FOR LOAD TRANSPORTATION: THE SOLVER TOOL AND ITS LIMITATIONS IN THE SEARCH FOR THE OPTIMAL SOLUTION}

\author{
Ricardo França Santos* E-mail: franca@casop.mar.mil.br \\ Eugênio Correa de Souza Junior* E-mail: eugelisa@bol.com.br \\ Marco Aurélio Carino Bouzada* E-mail: marco.bouzada@estacio.br \\ *Universidade Estácio de Sá, Rio de Janeiro, RJ
}

\begin{abstract}
Resumo: Este trabalho procura solucionar um problema característico de logística da Marinha do Brasil atinente à alocação, transporte e distribuição de gêneros frigorificados para as Organizações Militares da área do Grande Rio (RJ). Depois de uma breve revisão de literatura acerca da Programação Linear/Inteira e de algumas de suas aplicações, foi proposta a utilização da Programação Inteira, com o uso do suplemento Solver do Excel como ferramenta para obtenção da configuração ótima de carga da frota, ao menor custo de distribuição, visando ao atendimento da demanda programada. As premissas foram atendidas primeiramente em uma tentativa com uma planilha única, porém não foi possível encontrar uma solução convergente, sem problemas de degeneração e com tempo razoável de solução. Uma segunda solução foi proposta separando o problema em três fases, o que permitiu evidenciar as potencialidades e limitações da ferramenta Solver. Este trabalho mostrou a importância da formulação de um modelo realista e de uma análise crítica detalhada, o que pode ser constatado por meio da falta de convergência da primeira solução e com o sucesso alcançado pela segunda solução.
\end{abstract}

Palavras-chave: Pesquisa Operacional. Programação Inteira. Solver. Logística de Distribuição. Otimização de Carga.

Abstract: This work tries to solve a typical logistics problem of Navy of Brazil regards the allocation, transportation and distribution of genera refrigerated for Military Organizations within Grande Rio (RJ). After a brief review of literature on Linear/Integer Programming and some of their applications, we proposed the use of Integer Programming, using the Excel's Solver as a tool for obtaining the optimal load configuration for the fleet, obtaining the lower distribution costs in order to meet the demand schedule. The assumptions were met in a first attempt with a single spreadsheet, but it could not find a convergent solution, without degeneration problems and with a reasonable solution time. A second solution was proposed separating the problem into three phases, which allowed us to highlight the potential and limitations of the Solver tool. This study showed the importance of formulating a realistic model and of a detailed critical analysis, which could be seen through the lack of convergence of the first solution and the success achieved by the second one.

Key-words: Operational Research. Integer Programming. Solver. Distribution Logistics. Load Optimization. 


\section{INTRODUÇÃO}

A Marinha do Brasil realiza suas atividades de abastecimento por meio de um sistema que é constituído por órgãos de distribuição, processos e recursos de qualquer natureza, interligados e interdependentes, estruturado com a finalidade de prover gêneros necessários à manutenção das Organizações Militares (OM). Para isso conta com depósitos primários, depósitos secundários e depósitos regionais.

Dentre os depósitos regionais existe o Depósito de Subsistência da Marinha no Rio de Janeiro, que foi criado em 1955. O DepSubMRJ possui como missão executar as tarefas de receber, periciar, estocar, controlar e fornecer gêneros alimentícios, a fim de contribuir para a prontidão operativa dos Meios Navais, Aeronavais, Fuzileiros Navais e demais Organizações Militares da Marinha.

Em relação à função logística de transporte, diversos requisitos devem ser atendidos pelo DepSubMRJ, principalmente em relação à temperatura da carga e do veículo, assim como em relação a outras características deste último, como estado de conservação, de higiene e de segurança.

O DepSubMRJ dispõe de um número limitado de veículos de transporte de cargas que atendem a esses requisitos, que nem sempre estão disponíveis, devido a problemas mecânicos ou ausência de motoristas.

A frota de veículos é constituída de caminhões leves e ágeis que carregam cargas menores e caminhões pesados para cargas maiores em maiores distâncias. A distribuição da frota é realizada de modo empírico, baseada na experiência e competência dos profissionais, a partir das quais é produzida uma programação semanal, sustentada em fatores como demanda, localização do destinatário, condições de acesso, distância, disponibilidade do motorista e consumo de combustível, entre os principais fatores.

Por outro lado, existe uma demanda por parte das OM, no que tange aos gêneros frigorificados, que necessitam ser atendidos por essa frota. A programação semanal atende tanto às $\mathrm{OM}$ na cidade do Rio de Janeiro, quanto às $\mathrm{OM}$ das cidades próximas. As entregas são realizadas de segunda-feira a sexta-feira, inclusive feriados. Na tabela 1 a seguir, estão listados os custos fixos e variáveis da operação. 
Tabela 1 - Custos fixos e variáveis

\begin{tabular}{ll}
\hline Custos fixos (em $\mathbf{R} \$ \mathbf{m e ̂ s})$ & Custos variáveis (em $\mathbf{R} \mathbf{\$} \mathbf{K m})$ \\
\hline Depreciação do veículo $-13.500,00$ & Manutenção $-2,25$ \\
Licenciamento e seguro $-2.025,00$ & Combustível $-1,00$ \\
Reserva para seguro $-1.600,00$ & Lubrificação $-0,05$ \\
Salários (motorista e ajudante) $-22.500,00$ & Pneus $-0,20$ \\
\hline
\end{tabular}

Salários (motorista e ajudante) - 22.500,00 Pneus $-0,20$

Fonte: Elaboração própria

Na tabela 2 a seguir, estão registrados os valores mínimo e máximo de entrega diária.

Tabela 2 - Entrega mínima e máxima por dia (em toneladas)

\begin{tabular}{lccccc}
\hline & Segunda & Terça & Quarta & Quinta & Sexta \\
\hline Entrega Mínima & 31 & 25 & 37 & 29 & 13 \\
Entrega Máxima & 45 & 32 & 43 & 33 & 38 \\
\hline
\end{tabular}

Fonte: Elaboração própria

Na tabela 3 a seguir, podem ser observadas a distância e a demanda semanal de cada OM.

Tabela 3 - Distância e demanda semanal de cada OM

\begin{tabular}{lll}
\hline OM & Demanda Semanal (em ton) & Distância $(\mathrm{em} \mathrm{Km})$ \\
\hline Alfa & 8 & 12 \\
Bravo & 15 & 5 \\
Charlie & 16 & 140 \\
Delta & 18 & 160 \\
Echo & 20 & 15 \\
Foxtrot & 26 & 10 \\
Golf & 19 & 180 \\
Hotel & 14 & 06 \\
India & 16 & 25 \\
Juliet & 13 & 20 \\
\hline
\end{tabular}

Fonte: Elaboração própria

O transporte representa um significativo custo logístico por parte das empresas, absorvendo de um a dois terços do total. Segundo Ballou (2006), o transporte, quando é realizado por frota e equipamentos próprios, pode propiciar um melhor desempenho operacional, maior disponibilidade e capacidade de transporte e gerar custos menores. Isto geralmente é possível quando o volume de carga é elevado, sendo mais econômico possuir um serviço de transporte próprio do que contratá-lo.

Por outro lado, nem sempre isso é possível devido às exigências de requisitos especiais tais como entrega rápida com confiabilidade muito elevada, indisponibilidade de algum equipamento especial, manuseio especial da carga ou um serviço que deva estar disponível quando necessário. 
Neste trabalho, será considerada apenas a realização do serviço de transporte por frota própria, em que seus custos fixos e variáveis são perfeitamente conhecidos.

O gerente de tráfego de carga do DepSubMRJ é responsável pela decisão de utilização da frota, onde os objetivos de menores custos e melhor desempenho na entrega são perseguidos.

A montagem do plano de rota é um problema encontrado para direcionar os veículos através das diversas vias. Ballou (2008) menciona que o cálculo da rota pode ser feito pela mínima distância, mínimo tempo ou por uma combinação destes. Além disso, o custo total é dependente não só do custo do transporte, mas também do custo de estocagem.

Por outro lado, as empresas estocam produtos visando à melhoria da compensação entre oferta e demanda, além de diminuir seus custos totais. Neste sentido, os custos de armazenagem e manuseios de materiais podem ser justificados pela redução dos custos de transporte e de produção, pois os estoques podem reduzir os custos de transporte ao permitir que quantidades maiores e mais econômicas de carga sejam transportadas.

Neste trabalho, buscou-se responder a seguinte questão-problema: como buscar de forma determinística a configuração ótima de carga e de trajeto da frota de caminhões, de modo a se tentar obter o menor custo na distribuição de suprimentos da Marinha?

Como objetivo secundário, procurou-se apresentar subsídios para a discussão a respeito das limitações da ferramenta Solver do Excel no processo de busca da solução ótima para problemas dessa natureza.

Para tal, o referencial teórico apresentado a seguir revisa a abordagem que será usada para atacar o problema, ilustrando também alguns exemplos de aplicação. Em seguida, é descrito de forma mais específica como o problema em questão é abordado metodologicamente. Os resultados são, então, apresentados e discutidos, antes das considerações finais serem apresentadas. 


\section{REFERENCIAL TEÓRICO}

\section{1 Programação Linear}

Segundo Moore e Weatherford (2005), a programação linear (PL) tem sido bastante utilizada desde a década de 1940 pelos militares e até hoje, muitas empresas se beneficiam do seu uso. Segundo pesquisa da revista Fortune, 85\% das empresas já utilizaram ou utilizam programação linear. A PL teve como um de seus inventores George B. Dantzig, que descobriu o algoritmo Simplex, que é o mecanismo matemático utilizado para resolver os problemas da PL.

A programação linear então serviu de base para outras ferramentas gerenciais tais como a programação não-linear, a programação inteira e outras técnicas de otimização. Outro matemático, Leonid G. Khanchian descobriu o algoritmo de tempo polinomial que, teoricamente, é superior a algoritmos polinomiais como o algoritmo Simplex. Outros algoritmos mais rápidos foram descobertos mais tarde, como o algoritmo de Karmarkar (MOORE; WEATHERFORD, 2005).

De acordo com os autores, todo modelo de programação linear tem duas características importantes: uma função objetivo a ser maximizada ou minimizada e as restrições. Esse modelo de otimização restrito fornece meios de solucionar problemas em que os recursos são escassos de modo a otimizar um objetivo de interesse.

Segundo Colin (2007, p.28) o algoritmo Simplex utiliza uma série de definições e conceitos associados entre os quais a definição de solução básica, o conceito de variável básica e não-básica e a definição de solução básica viável. O autor define uma solução viável como sendo aquela que pode ser implementada considerando que os valores das variáveis de decisão estão de acordo com as restrições.

De acordo com o mesmo, o algoritmo Simplex é o mesmo algoritmo utilizado nos softwares específicos para a solução de problemas de PL e caminha de uma solução viável para outra, fazendo com que o valor da função-objetivo seja diminuído até o ponto ótimo ser alcançado. Esse algoritmo é simples e resolve uma gama de problemas e modelos do mundo real, gerando uma grande economia de recursos. 
Ainda segundo o mesmo, para $m$ equações e $n$ variáveis existem no máximo $n ! / m !(n-m)$ ! soluções básicas. Ainda assim, o método Simplex geralmente converge para a solução ótima em aproximadamente $3 \mathrm{~m} / 2$ iterações.

De acordo com Colin (2007), em relação às restrições da utilização do algoritmo Simplex, são conhecidos os problemas de escala quando existem muitos cálculos numéricos e a variabilidade entre o maior e o menor número é muito grande, causando problemas de cálculo numérico.

Outros problemas dizem respeito às: (i) soluções inexistentes - quando não há solução que satisfaça as restrições; (ii) soluções ilimitadas - quando o algoritmo não consegue encontrar um ponto de máximo em um problema de maximização ou de mínimo num problema de minimização; e (iii) soluções múltiplas - quando mais de uma solução satisfaz todas as restrições e alcança um valor extremo da funçãoobjetivo (COLIN, 2007).

O mesmo autor ainda alega que o algoritmo Simplex não resolve qualquer problema de PL. Quando todas as variáveis são estritamente positivas nas soluções básicas viáveis, estamos diante de um problema que é conhecido como nãodegenerado.

Em outras palavras, se uma ou mais das variáveis básicas numa solução têm o valor zero, a solução é chamada de solução básica degenerada. A degeneração traz consigo problemas de convergência do algoritmo, sendo difícil identificar a solução ótima. Geralmente, os softwares utilizados no mercado, tais como o Solver, já tratam dessas particularidades, de forma invisível para o usuário (COLIN, 2007).

\subsection{Programação Inteira}

A Programação Inteira (PI) surgiu como uma limitação da PL, quando na resolução dos problemas havia a necessidade de uso de variáveis inteiras. O nível de dificuldade para resolução de problemas de PI é similar à resolução de problemas com PL, porém enquanto estas possuem um espaço infinito de soluções, aquelas possuem um espaço finito de soluções, o que implica que a solução de PL é mais fácil que a solução de PI (COLIN, 2007). 


\section{3 Pesquisas envolvendo Programação Linear/Inteira}

Alguns trabalhos foram resolvidos com a utilização da PL, entre os quais se encontra o clássico problema do caixeiro-viajante. Outros problemas foram apresentados mostrando a importância da função logística de transporte. Moore e Weatherford (2005) apresentam um exemplo de problema relacionado ao transporte atinente à maximização do lucro considerando o transporte de mercadorias enviadas por duas fábricas.

Outro trabalho relevante foi o trabalho de Mine et al. (2009), que sugere um algoritmo híbrido para o problema de roteamento de veículos com entrega e coleta simultânea (PRVCES).

Farias (2009) apresenta um problema que envolve cinco propostas de projeto, cada uma com uma expectativa de se tornar um contrato, do qual se deseja tomar a decisão de quais dessas propostas deveriam ser implementadas utilizando os recursos concorrentes da empresa. Segundo o autor, se for considerado o problema de forma inteira (em particular, binária), não será considerado o risco envolvido nos projetos. Porém, no mundo real, as incertezas existem e o problema não deveria ser tratado pela abordagem determinística.

Carvalho et al. (2000) procuram otimizar o uso da água no perímetro irrigado. Os autores propuseram o uso da técnica de programação linear com um modelo cuja função-objetivo visava maximizar as receitas mensais em função da área cultivada com determinadas culturas, tendo como restrições a área e a disponibilidade de água.

Nogueira et al. (2006) apresentam um exemplo de aplicação da programação inteira onde o contador responsável por um escritório de perícias deve decidir sobre os honorários a serem cobrados. Os autores concluem que a utilização deste método pode contribuir na otimização de dois fatores relevantes que afetam a remuneração do trabalho pericial: o prazo de recebimento e 0 índice de remuneração. 


\section{METODOLOGIA}

\subsection{Objeto do estudo}

Atualmente, a distribuição da frota é realizada de modo empírico, baseada na experiência e competência dos profissionais envolvidos nessa tarefa. O problema desta pesquisa consiste em programar, de forma eficaz, a alocação de veículos frigoríficos do DepSubMRJ, atendendo às demandas semanais e minimizando os custos de distribuição. Existe uma programação semanal que atende tanto às $\mathrm{OM}$ na cidade do Rio de Janeiro, quanto às cidades próximas. As entregas são realizadas de segunda-feira a sexta-feira, inclusive feriados.

Como restrições há uma programação de demanda e percursos em distância a percorrer para atender cada OM. A frota de veículos frigoríficos é constituída por caminhões de capacidades diferenciadas ( 14 ton, 08 ton e 03 ton), o que caracteriza a capacidade e a agilidade do caminhão no percurso. Os custos fixos e variáveis do transporte, a demanda semanal de cada OM e o valor máximo e mínimo de entrega diária foram levantados junto à instituição, e foram apresentados nas tabelas 1, 2 e 3 anteriores.

\subsection{Modelagem e tratamento dos dados}

Para resolver a questão proposta foi utilizada a ferramenta Solver do Excel, buscando-se obter a programação que importasse em menor custo possível. Como primeiro passo foi necessário identificar quais eram os fatores/variáveis que influenciavam diretamente no custo envolvido na distribuição de suprimentos da Marinha. Fruto desta primeira análise chegou-se, então, a algumas premissas.

A primeira delas diz respeito à composição do custo total, que engloba o custo fixo, que não se altera, e o custo variável, que sofre influência de acordo com a programação que for executada. Neste caso, para diminuir o custo total, o que importa é fazer a distribuição nas OM com a menor quilometragem possível, pois é isso que influencia diretamente o custo variável.

$O$ segundo pressuposto indica que para se obter menor quilometragem deve haver o mínimo de viaturas possível deslocando-se para as OM, a fim de satisfazer 
suas necessidades. No caso em que haja a opção de suprir determinada OM, empregando uma ou duas viaturas, deve-se escolher a primeira alternativa, pois a segunda implica no dobro da quilometragem (um percurso por cada viatura).

A terceira premissa preconiza que é desejável suprir as OM de uma só vez, pois quanto mais viagens forem realizadas, maior será a quilometragem. As melhores escolhas são, portanto, aquelas em que se utiliza apenas um dia para suprir determinada OM.

Finalmente, deve-se observar que embora não haja diferença no cálculo dos custos variáveis, deve haver penalização para a alocação de viaturas com pouca carga. Afinal, quando isto ocorre, está se deixando de aproveitar espaço útil nas viaturas, o que não deixa de ser uma perda, ainda que implícita.

Foi realizada a programação no Excel, procurando-se encontrar uma reposta determinística para a questão de estudo, como alternativa para a programação da frota, executada apenas com base na experiência dos profissionais da Marinha.

A metodologia utilizada durante o processo é composta de três fases ou etapas para a solução do problema. Em todas as fases, a modelagem envolveu a seguinte sistemática com utilização da ferramenta Solver: 1) Definir a variável-objetivo; 2) Definir as variáveis de decisão; 3) construir a função-objetivo e 4) definir as restrições.

$\mathrm{Na}$ primeira etapa, para atendimento da modelagem geral definida, foram desenvolvidas as seguintes atividades:

Foi realizada a escolha de uma célula de destino do Solver que priorizasse a programação considerando o menor custo possível, ou seja, a menor quilometragem possível com a menor ociosidade possível (definir a variável-objetivo).

Para tal, foram definidas como variáveis de decisão a tonelagem alocada a cada uma das nove viaturas em cada dia da semana.

$\mathrm{Na}$ construção da função-objetivo, foi estimada a demanda atendida para cada OM e para cada dia da semana, por meio do somatório da divisão da demanda alocada pela capacidade de cada viatura considerada (utilizou-se a função do Excel "arredondar para cima"), sendo seu resultado multiplicado pela distância em Km e esse valor fornecido como resultado final de cada OM.

Em relação às restrições, foram inseridos na planilha do Excel, para utilização do Solver: o somatório do total das demandas estimadas calculadas por OM e por Revista Produção Online, Florianópolis, SC, v.12, n. 1, p. 185-204, jan./mar. 2012. 
dia da semana como sendo igual aos valores fornecidos das demandas por OM (OM Alfa=8, OM Bravo=15, etc.); as restrições das células da tabela como sendo inteiras para cada um dos valores da tabela geral construída no passo dois e para cada dia da semana; e o somatório das demandas atendidas por viatura para todas as OM como sendo menor ou igual à demanda semanal estimada fornecida por OM.

Na segunda etapa, foi criada uma planilha geral contendo todos os tipos de viatura (4 de 14T, 3 de $8 \mathrm{~T}$ e 2 de 3T), as distâncias percorridas por OM, as demandas estimadas e as entregas calculadas por OM e por dia da semana e o custo total calculado. Foi executado um Solver geral utilizando resultados de Solvers intermediários rodados em planilhas distintas contendo as demandas calculadas para cada OM, levando-se em consideração as diferenças entre as capacidades de carga das viaturas e o seu não preenchimento total nas viagens.

$\mathrm{Na}$ planilha geral, foi calculado também, com o auxílio das funcionalidades do Excel, o custo total para as viaturas, considerando os custos fixos e variáveis, sendo estes calculados em função da quilometragem percorrida para atender a programação por OM. Esses valores foram obtidos das planilhas intermediárias.

Na planilha geral, foi considerada como variável-objetivo o valor máximo obtido da média das entregas realizadas nas OM em cada um dos dias da semana, conseguidas por meio dos valores das planilhas intermediárias, considerando o somatório do produto dos dias da semana pela demanda de cada OM.

Foram consideradas como restrições: que a capacidade calculada das viaturas em uso fosse menor ou igual à capacidade real das respectivas viaturas; que as células possuíssem valores numéricos; e que existisse pelo menos uma viatura que atendesse a OM em um dia da semana considerado.

Nas planilhas em que foram rodados Solvers intermediários, a variável-objetivo foi obtida como sendo a capacidade mínima (menor valor) da soma da sobra ou resto (diferença entre a capacidade utilizada pela viatura e a capacidade estimada) com o somatório do produto entre a distância percorrida e as capacidades das viaturas utilizadas pela OM, estes últimos tendo como valores iniciais da planilha os valores encontrados na primeira etapa.

Foram utilizadas como restrições as quantidades disponíveis de viaturas (4 viaturas de 14T, 3 viaturas de $8 \mathrm{~T}$ e duas viaturas de $3 \mathrm{~T}$ ), as restrições numéricas para as células e a consideração sobre a sobra ou resto, ou seja, que a capacidade Revista Produção Online, Florianópolis, SC, v.12, n. 1, p. 185-204, jan./mar. 2012. 
de utilização da viatura fosse maior ou igual à demanda estimada para aquela viatura.

Na última etapa, foi utilizado o Solver para consolidar as planilhas contendo Solvers individualizados para cada dia da semana.

Nos Solvers individualizados das planilhas por dia da semana, foi considerado como variável-objetivo o somatório da sobra com a distância total. A distância total foi representada pela soma do produto entre a distância estimada e as quantidades dos tipos de viaturas utilizados. Isto visava ao atendimento do princípio de menor quilometragem possível e menor capacidade ociosa possível.

Em relação às restrições, foi definido que o número de viaturas de uma determinada capacidade utilizada teria que ser menor ou igual à quantidade de viaturas desse tipo disponível para uso. Além da restrição obrigatória da célula ser numérica, a outra restrição utilizada foi em relação ao somatório da demanda atendida pelas $\mathrm{OM}$, que teria que ser maior ou igual ao somatório da demanda semanal necessária para essas OM.

\subsection{Limitações}

Há de se mencionar as limitações naturais decorrentes da necessidade de os parâmetros utilizados nas otimizações estarem corretos. Tal observação se faz mais necessária no caso da demanda, que costuma apresentar um caráter probabilístico, mas que aqui foi tratada de forma determinística.

Além disso, o procedimento de 3 fases utilizado para a solução do problema devido à limitação da ferramenta Solver - pode ser considerado uma heurística. Em vista disso, o caráter ótimo da solução encontrada deve ser encarado com ressalvas, antes de uma constatação ser realizada.

\section{APRESENTAÇÃO E DISCUSSÃO DOS RESULTADOS}

\subsection{Primeira tentativa de solução}

Baseando-se nas premissas listadas anteriormente, buscou-se inicialmente modelar o problema no Excel, criando uma tabela geral de variáveis ajustáveis, 
utilizando números inteiros, correspondendo à tonelagem alocada em cada viatura. Estas variáveis indicariam, diariamente, que viaturas seriam empregadas, qual a capacidade utilizada das viaturas e que OM seriam abastecidas.

Para um custo mínimo, ou seja, menor quilometragem, foram estabelecidas algumas restrições. Em primeiro lugar, diariamente, cada viatura não poderia ser abastecida com uma tonelagem acima de sua capacidade. Era imperativo também que as demandas de cada OM deveriam ser satisfeitas, ao término da semana. Por fim, as variáveis de decisão deveriam ser binárias.

A tabela 4 a seguir traz a configuração do problema para a segunda-feira, considerando que para cada dia da semana o número de células ajustáveis era a mesma. Desta forma, o valor 0 significaria a não utilização da viatura, ao passo que o valor 1 indicaria o uso do veículo.

Tabela 4 - Extrato das células variáveis da tabela geral

\begin{tabular}{|c|c|c|c|c|c|c|c|c|c|}
\hline & & & & & Segunc & & & & \\
\hline & OM A & OM B & OM C & & OM D & OM E & OM F & OM G & \\
\hline $\operatorname{Vtr} 1(14 \mathrm{~T})$ & & 0 & 0 & 0 & & 0 & 0 & 0 & 0 \\
\hline $\mathrm{Vtr} 2(14 \mathrm{~T})$ & & 0 & 0 & 0 & & 0 & 0 & 0 & 0 \\
\hline $\operatorname{Vtr} 3(14 \mathrm{~T})$ & & 0 & 0 & 0 & & 0 & 0 & 0 & 0 \\
\hline $\operatorname{Vtr} 4(14 \mathrm{~T})$ & & 0 & 0 & 0 & & 0 & 0 & 0 & 0 \\
\hline $\operatorname{Vtr} 5(8 \mathrm{t})$ & & 0 & 0 & 0 & & 0 & 0 & 0 & 0 \\
\hline$V \operatorname{tr} 6(8 t)$ & & 0 & 0 & 0 & & 0 & 0 & 0 & 0 \\
\hline$V \operatorname{tr} 7(8 \mathrm{t})$ & & 0 & 0 & 0 & & 0 & 0 & 0 & 0 \\
\hline $\operatorname{Vtr} 8(3 \mathrm{t})$ & & 0 & 0 & 0 & & 0 & 0 & 0 & 0 \\
\hline $\operatorname{Vtr} 9(3 t)$ & & 0 & 0 & 0 & & 0 & 0 & 0 & 0 \\
\hline Totais & & 0 & 0 & 0 & & 0 & 0 & 0 & 0 \\
\hline
\end{tabular}

Fonte: Elaboração própria

Apesar da configuração do problema obedecer a uma lógica correta, ao se utilizar a ferramenta Solver do Excel, não foi possível se chegar a uma solução, sendo exposta a seguinte mensagem: "Excesso de células ajustáveis". Sem dúvida, haveria grande necessidade de processamento dos cálculos para se testar todas as variáveis na tabela geral. Por este motivo, esta que seria a solução mais direta no Solver teve que ser descartada.

Em diversas outras tentativas de resolução do problema, as limitações de cálculo do Solver ficaram evidentes. Existiram casos em que o programa demorava horas sem que apresentasse uma solução. Fruto destas experiências, chegou-se à

Revista Produção Online, Florianópolis, SC, v.12, n. 1, p. 185-204, jan./mar. 2012. 
conclusão de que a modelagem do problema deveria considerar fases de resolução que não excedessem a capacidade da ferramenta empregada.

\subsection{Primeira solução}

Levando-se em consideração as premissas levantadas e a conclusão da necessidade de faseamento, anteriormente mencionada, chegou-se à primeira solução determinística com o uso da ferramenta Solver no Excel. Para essa primeira modelagem válida, alguns procedimentos foram adotados. Em primeiro lugar, foi criada uma planilha com parâmetros, para servir como base para as demais planilhas que seriam criadas. Além disso, a solução foi dividida em duas etapas, sendo empregada a ferramenta Solver em cada uma destas etapas.

$\mathrm{Na}$ primeira etapa, para cada OM foi realizado um modelo Solver individual, com o propósito de verificar qual seria a melhor alocação de viaturas para satisfazer a demanda de uma única vez. A célula objetivo priorizou a menor quilometragem possível, com a menor ociosidade possível. Por este motivo, sua composição foi de viaturas empregadas, multiplicada pela distância, mais o espaço ocioso. Com isto, procurou-se tentar atender as restrições pré-estabelecidas.

$\mathrm{Na}$ segunda etapa, foi executado um Solver geral para decidir quais as OM que seriam atendidas em cada dia da semana. Para tal, tomou-se como base o resultado da etapa anterior, que alocou a melhor quantidade de viaturas para satisfazer as OM de uma única vez. Neste Solver foram impostas as restrições de quantidade de viatura e de que cada OM não poderia ser atendida mais de uma vez na semana. $\mathrm{O}$ mais importante na segunda etapa foi satisfazer as restrições.

Por isso, a média foi um artifício utilizado para criar a variável objetivo; afinal, qualquer solução apresentaria o mesmo custo, pois a quilometragem seria sempre a mesma. Inicialmente, a variável padrão foi definida, tomando como base o desvio padrão, mais isto dificultou o trabalho do Excel para apresentar uma solução válida.

Cabe ressaltar que não foram utilizados números necessariamente binários para as variáveis de decisão na segunda etapa. No entanto, na realidade, os números sempre eram 0 ou 1 , devido à restrição de se atender a $\mathrm{OM}$ apenas uma vez na semana. As tabelas 5, 6 e 7 a seguir trazem o detalhamento de uma das configurações válidas encontradas, nesta primeira solução determinística. 
Tabela 5 - OM atendidas por dia

\begin{tabular}{lrrrrr}
\hline OM & Seg & Ter & Qua & Qui & \multicolumn{2}{c}{ Sex } \\
\hline Alfa & 0 & 1 & 0 & 0 & 0 \\
Bravo & 0 & 0 & 1 & 0 & 0 \\
Charlie & 0 & 1 & 0 & 0 & 0 \\
Delta & 1 & 0 & 0 & 0 & 0 \\
Echo & 1 & 0 & 0 & 0 & 0 \\
Foxtrot & 0 & 1 & 0 & 0 & 0 \\
Golf & 1 & 0 & 0 & 0 & 0 \\
Hotel & 1 & 0 & 0 & 0 & 0 \\
India & 0 & 0 & 0 & 0 & 1 \\
Juliet & 0 & 0 & 0 & 1 & 0 \\
Entrega & 71 & 50 & 15 & 13 & 16 \\
\hline
\end{tabular}

Fonte: Elaboração própria

Tabela 6 - Uso de viaturas

\begin{tabular}{lllllll}
\hline Uso Vtr & Seg & Ter & Qua & Qui & \multicolumn{2}{c}{ Sex } \\
\hline Vtr 14 Ton & 4 & 2 & 0 & 1 & 0 \\
Vtr 8 ton & 3 & 3 & 2 & 0 & 2 \\
Vtr 3 Ton & 0 & 0 & 0 & 0 & 0 \\
\hline
\end{tabular}

Fonte: Elaboração própria

Tabela 7 - Distribuição de viaturas e quilometragens envolvidas

\begin{tabular}{|c|c|c|c|c|c|c|c|}
\hline OM & $\begin{array}{l}\text { Vtr } 14 \\
\text { Ton }\end{array}$ & $\operatorname{Vtr} 8$ ton & $\begin{array}{l}\text { Vtr } 3 \\
\text { Ton }\end{array}$ & & Demanda & Distância & $\begin{array}{l}\text { Km por } \\
\text { OM }\end{array}$ \\
\hline Alfa & & 0 & I & 0 & 8 & 12 & 12 \\
\hline Bravo & & 0 & $?$ & 0 & 15 & 5 & 10 \\
\hline Charlie & & 0 & $?$ & 0 & 16 & 140 & 280 \\
\hline Delta & & 1 & 1 & 0 & 18 & 160 & 320 \\
\hline Echo & & 1 & 1 & 0 & 20 & 15 & 30 \\
\hline Foxtrot & & 2 & ) & 0 & 26 & 10 & 20 \\
\hline Golf & & 1 & I & 0 & 19 & 180 & 360 \\
\hline Hotel & & 1 & ) & 0 & 14 & 6 & 6 \\
\hline India & & 0 & $?$ & 0 & 16 & 25 & 50 \\
\hline Juliet & & 1 & ) & 0 & 13 & 20 & 20 \\
\hline Total & & & & & & & 1108 \\
\hline
\end{tabular}

Fonte: Elaboração própria

\subsection{Segunda solução}

A primeira solução não satisfazia o requisito de entrega máxima e mínima imposta pelo problema. Embora isto não fosse um prejuízo considerável, por não 
implicar em maiores custos ou indisponibilidade de viatura, surgiu a necessidade de se elaborar uma segunda solução, que satisfizesse a todos os requisitos.

Cabe ressaltar que a segunda solução é mais trabalhosa que a primeira, embora seja a mais completa e a única que realmente satisfaz o problema proposto. Foram, portanto, adotados alguns procedimentos. Em primeiro lugar, foi criada uma planilha de parâmetros, de forma semelhante à anterior. Em seguida, dividiu-se a resolução em três fases.

Para a primeira fase, partiu-se da premissa de que a melhor coisa a ser feita é suprir o máximo de OMs o mais rápido possível, obedecendo, é claro, as restrições de entrega máxima e mínima. Além disso, a OM deve ser suprida em apenas um dia, para minimizar a quilometragem. Por este motivo, foi criado um Solver diário, para decidir quais OM seriam supridas diariamente.

A variável-objetivo foi a sobra de toneladas a serem supridas, devendo ser a mínima possível. Cabe ressaltar que as planilhas estavam relacionadas de forma que a demanda considerada em determinado dia fosse igual à demanda que deixou de ser atendida no dia anterior, de forma a não se atender mais de uma vez uma determinada localidade. A tabela 8 a seguir traz o resultado do Solver para segundafeira.

\begin{tabular}{lrrrr}
\multicolumn{5}{l}{ Tabela 8 - Primeira fase (segunda-feira) } \\
\hline Entrega (máx) & 45 & 45 & & \\
Entrega (mín) & 31 & 45 & Efetuada & Resta \\
\hline Alfa & 8 & 0 & 0 & 8 \\
Bravo & 15 & 0 & 0 & 15 \\
Charlie & 16 & 0 & 0 & 16 \\
Delta & 18 & 0 & 0 & 18 \\
Echo & 20 & 0 & 0 & 20 \\
Foxtrot & 26 & 1 & 26 & 0 \\
Golf & 19 & 1 & 19 & 0 \\
Hotel & 14 & 0 & 0 & 14 \\
India & 16 & 0 & 0 & 16 \\
Juliet & 13 & 0 & 0 & 13 \\
Total & 165 & & & 120 \\
\hline Fotre: Ela & & & &
\end{tabular}

Fonte: Elaboração própria

Com os dados da primeira fase, chegou-se a uma tabela que demonstrava como as OM seriam atendidas, ao longo da semana, conforme exposto na Tabela 9 a seguir. Faltava, no entanto, determinar como fazer a distribuição das viaturas para 
que fosse atendido o objetivo maior do problema: obter a melhor programação para reduzir custos. Para tal, foi realizada a segunda fase.

Como base para a segunda fase, além da tabela 9, foram adotados, na variável-objetivo, os princípios de menor quilometragem possível e também o de menor capacidade ociosa possível (espaço vazio em viatura utilizada). Estes são os mesmos parâmetros que foram utilizados na solução anterior.

Tabela 9 - Consolidação da primeira fase

\begin{tabular}{lrrrrrrr}
\hline OM & Seg & Ter & Qua & Qui & Sex & \multicolumn{2}{c}{ Dist } \\
\hline Alfa & 0 & 0 & 1 & 0 & 0 & 12 \\
Bravo & 0 & 0 & 1 & 0 & 0 & 5 \\
Charlie & 0 & 0 & 0 & 1 & 0 & 140 \\
Delta & 0 & 1 & 0 & 0 & 0 & 160 \\
Echo & 0 & 0 & 1 & 0 & 0 & 15 \\
Foxtrot & 1 & 0 & 0 & 0 & 0 & 10 \\
Golf & 1 & 0 & 0 & 0 & 0 & 180 \\
Hotel & 0 & 1 & 0 & 0 & 0 & 6 \\
India & 0 & 0 & 0 & 1 & 0 & 25 \\
Juliet & 0 & 0 & 0 & 0 & 1 & 20 \\
Totais & 45 & 32 & 43 & 32 & 13 & \\
\hline
\end{tabular}

Fonte: Elaboração própria

As restrições adotadas em cada Solver desta segunda fase disseram respeito, principalmente, à limitação de viatura. A tabela 10 a seguir traz o resultado do Solver de segunda-feira, nesta segunda fase. Nesta tabela, "Dem at" refere-se à capacidade das viaturas empregadas, enquanto que "Dem ref" diz respeito à necessidade das OM.

Na última fase desta resolução, foi feita apenas a elaboração de uma planilha de consolidação, com o resultado final da primeira e segunda fases da resolução. A terceira fase detalha, portanto, diariamente, o percurso das viaturas que serão empregadas na distribuição de suprimentos pelas OM.

\subsection{Comparação entre as soluções}

A única vantagem da primeira solução em relação à segunda é ser relativamente mais simples, exigindo menos tabelas Solver. Esta aparente vantagem, no entanto, talvez nem mesmo possa ser considerada, tendo em vista 
que somente na segunda solução foram atendidos os requisitos propostos pelo problema de entrega máxima e mínima.

Tabela 10 - Segunda fase (segunda-feira)

\begin{tabular}{rrrrrrrr}
\hline & \multicolumn{7}{c}{ Dist envo } \\
\hline & 14 & 8 & 3 & & Demanda & Dem at & \multicolumn{2}{c}{ Dem ref } \\
& 0 & 0 & 0 & 0 & 8 & 0 & 0 \\
& 0 & 0 & 0 & 0 & 15 & 0 & 0 \\
& 0 & 0 & 0 & 0 & 16 & 0 & 0 \\
& 0 & 0 & 0 & 0 & 18 & 0 & 0 \\
& 0 & 0 & 0 & 0 & 20 & 0 & 0 \\
& 2 & 0 & 0 & 20 & 26 & 28 & 26 \\
& 1 & 1 & 0 & 360 & 19 & 22 & 19 \\
& 0 & 0 & 0 & 0 & 14 & 0 & 0 \\
& 0 & 0 & 0 & 0 & 16 & 0 & 0 \\
& 0 & 0 & 0 & 0 & 13 & 0 & 0 \\
Utl & 3 & 1 & $0<=$ & & Cap total & 50 & 45 \\
Disp & 4 & 3 & 2 & & & &
\end{tabular}

Fonte: Elaboração própria

Além disso, a segunda solução utiliza de forma mais eficaz o espaço das viaturas. Isto acontece porque ela não considera as OM de forma isolada, e sim a entrega que deve ser efetuada no dia. Evita-se, com isso, a sobreposição de sobras de espaços vazios nas viaturas quando estas são alocadas considerando-se as OM de forma isolada. Como resultado disso, há inclusive, a utilização de viaturas de 3 toneladas, o que não ocorria na primeira solução.

Finalmente, pode-se dizer que há maior probabilidade de a segunda solução ser a ótima. Ela está, desta forma, em consonância com o objetivo maior da ferramenta Solver, que é apontar a melhor solução. No caso da primeira solução, há mais chances de haver alternativas melhores, e se assim for, o instrumento de apoio à decisão pode estar comprometido. Por isso, embora mais trabalhosa, a segunda solução é a que deve ser considerada na solução do problema proposto.

Pode-se constatar que a formulação de um bom modelo é crucial para a sua solução e que é importante a análise crítica detalhada para avaliação da real necessidade de se modelar uma variável como inteira. Tal constatação foi baseada na falta de convergência do resultado na primeira solução e no sucesso alcançado com a nova modelagem na segunda solução, considerando a defasagem proposta. 
Cabe considerar que, embora possa ser trabalhosa a montagem do problema, após isto, com os dados correlacionados na planilha, não existem dificuldades para se efetuar novas soluções a partir de modificações que venham a ocorrer. No caso, por exemplo, de se acrescerem viaturas, ou haver aumento de demanda, a partir da mudança na planilha de parâmetros, todos os demais cálculos serão efetuados de forma automática. A solução apresentada não serve, por isso, apenas para responder ao momento atual, mas pode ser utilizada também em ocasiões futuras.

No processo de modelagem e otimização, a primeira etapa costuma ser a mais difícil. Na verdade, modelar um problema complexo consiste bem mais em arte do que ciência. Sim, é possível seguir diretrizes básicas e um roteiro genérico, mas, principalmente nos casos em que houver uma boa dose de complexidade presente no problema real, muito provavelmente será necessário fazer um bom uso da criatividade do modelador de forma que os objetivos estabelecidos sejam alcançados.

Tais constatações ficaram evidentes no processo de confecção do problema aqui abordado.

\section{CONCLUSÕES}

A resolução do problema real com o uso do Excel permitiu que se chegasse a uma visão acerca das potencialidades e das limitações da ferramenta Solver. Verificou-se neste sentido, que existe a necessidade de executar a modelagem do problema, considerando o processamento eletrônico que possui limitações. No entanto, após o faseamento das ações, a ferramenta Solver pode ajudar a resolver questões complexas, como a considerada neste trabalho.

Em relação às limitações da ferramenta Solver reportados na literatura, tomouse cuidado em relação ao problema mencionado de escala para que não houvesse muita variabilidade entre o maior e o menor número nos cálculos efetuados, o que poderia gerar problemas de convergência (COLIN, 2007).

Outra limitação da ferramenta Solver que deve ser mencionada diz respeito ao problema da degeneração. Conforme reportado na literatura (COLIN, 2007), se uma ou mais das variáveis básicas numa solução tem o valor zero, a solução é chamada de solução básica degenerada, e isto traz consigo problemas de convergência do 
algoritmo, sendo difícil identificar a solução ótima. Ainda que o algoritmo Simplex trate esses problemas, este fator deve ser considerado na tentativa de resolução na primeira solução.

Entretanto, na primeira solução, para atender os requisitos pré-estabelecidos e apresentar um valor para a célula alvo que fosse ótima, o Solver apresentou inicialmente, em alguns casos, uma demora excessiva. Conforme reportado na literatura (COLIN, 2007), para $m$ equações e $n$ variáveis, deveria haver, no máximo, $n ! / m !(n-m)$ ! soluções básicas e o algoritmo deveria convergir para a solução ótima em aproximadamente $3 \mathrm{~m} / 2$ iterações.

Trazendo essas fórmulas para o problema em questão, verifica-se que, no primeiro caso, existiam 362 equações relacionadas às restrições e 315 variáveis, o que poderia levar a 543 iterações, explicando a demora na solução e a não convergência para um valor ótimo. Por outro lado, com a solução vislumbrada utilizando-se a defasagem dos dias da semana, conseguiu-se uma convergência com 18 iterações.

Como indicação para pesquisas futuras, fica a sugestão de se tentar modelar tais problemas de forma ainda mais realista, considerando todos os aspectos mais importantes em relação à situação atacada.

Por exemplo, a demanda de cada OM poderia ser considerada de forma probabilística, através de uma distribuição de probabilidades condizente com o seu histórico de comportamento, o que estaria mais de acordo com a realidade. Isso seria possível por meio do uso de uma abordagem probabilística, como a Simulação de Monte Carlo (HERTZ, 1980; PAULA et al., 2007), ao invés da Programação Inteira, utilizada neste trabalho, que considerou a demanda de forma puramente determinística.

Cabe ressaltar, no entanto, que, no caso de modelagens ainda mais realistas (e, portanto, potencialmente mais complexas), tornam-se ainda mais importantes os cuidados em relação à modelagem mencionados nestas considerações finais, para que o esforço computacional na busca da solução ótima não ultrapasse os limites da viabilidade operacional.

Finalmente, vale sugerir que as limitações da ferramenta Solver levantadas ao longo do artigo sejam contornadas através da utilização de um otimizador profissional, mais poderoso, porém de acesso mais complicado. Tal sugestão 
poderia lidar com a limitação da falta de certeza quanto à obtenção de uma solução ótima para o problema.

\section{REFERÊNCIAS}

BALLOU, R. Logística empresarial. São Paulo: Atlas, 2008.

. Gerenciamento da cadeia de suprimentos/logística empresarial.

Porto Alegre: Bookman, 2006.

CARVALHO, D.; SOARES, A.; RIBEIRO, C.; SEDIYAMA, G.; PRUSKI, F.

Otimização do Uso da Água no Perímetro Irrigado do Gorutuba, Utilizando-se a Técnica da Programação Linear. RBEAA, v.4, n.2, p.203-209, 2000.

COLIN, E. Pesquisa operacional. Rio de Janeiro: LTC, 2007.

FARIAS, C. Abordando probabilisticamente um problema com decisões "sim ou não": um estudo para determinação de mix de projetos de consultoria.

Dissertação (Mestrado em Administração e Desenvolvimento Empresarial). Rio de Janeiro: UNESA, 2009.

HERTZ, D. Análise de risco em investimentos de capital. Biblioteca Harvard de Administração de Empresas, v.8, n.3, p. 1-14, 1980.

MINE, M. ; SILVIA, M.; OCHI, L.; SOUZA, M. Um algoritmo heurístico híbrido para o problema de roteamento de veículos com entrega e coleta simultânea. In: Encontro Nacional de Engenharia de Produção, 29., 2009, Salvador. Anais... Salvador:

ENEGEP, 2009.

MOORE, J.; WEATHERFORD, L. Tomada de decisão em administração com planilhas eletrônicas. Porto Alegre: Bookman, 2005.

NOGUEIRA, M.; PELEIAS, I.; PARISI, C.; ORNELAS, M. Otimização do mix operacional de um escritório de perícias: uma aplicação de programação linear. In: Seminários de Administração da FEA/USP, 9., 2006, São Paulo. Anais... São Paulo: SEMEAD, 2006.

PAULA, R.; CAPELO Jr, E.; COSTA, C. O Cálculo do Valor Presente Líquido com Tratamento do Risco através do Método de Simulação de Monte Carlo. In: Encontro da ANPAD, 31, 2007, Rio de Janeiro. Anais... Rio de Janeiro: ENANPAD, 2007.

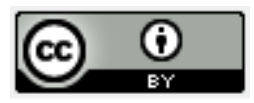

Artigo recebido em 18/11/2010 e aceito para publicação em 24/10/2011. 